\title{
Atomic Ground State Configurations of the Elements E159 and E160*
}

\author{
13. LiRICKE AND J. T. Waber

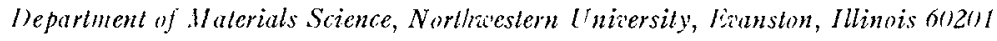

(Received 15 Octoher 1971)

Since the nuclear physicists have predicted an island of nuclear stabilit $y^{1}$ near element E164 with possible lifetimes of seconds or even years, the properties of elements with nearby atomic numbers have also become interesting from a chemical point of view. Various self-consistent atomic calculations have already been used to investigate ${ }^{2-7}$ the electronic ground state con- figurations, ionization potentials, radii and chemical properties of these elements up to element E172.

The latest nuclear calculations ${ }^{8}$ indicate that the most stable elements can be expected between E156 and E160. We have re-examined these elements br making a series of Dirac-Slater SCF calculations ${ }^{9}$ using $\frac{2}{3}$ of Slater's exchange term and the method of Snow 
TABLE I. The total energies, $L_{T}$, of the lowest lying electron configurations of elements E159 and E160.

\begin{tabular}{ccccccc}
\hline & \multicolumn{3}{c}{ Configuration $^{2}$} & & \\
Element & $7 d_{3 / 2}$ & $7 d_{5 / 2}$ & $9 s$ & $\begin{array}{c}N_{r} \\
(\mathrm{Ry})\end{array}$ & \multicolumn{1}{c}{$\begin{array}{c}\text { Difference } \\
(\mathrm{Ry})\end{array}$} \\
\cline { 2 - 7 } E159 & 4 & $\ldots$ & 1 & 312 & 362.365 & \multicolumn{1}{l}{0.0} \\
& 4 & 1 & $\ldots$ & 62.302 & 0.063 \\
E160 & 4 & 1 & 1 & 321 & 458.395 & 0.0 \\
& 4 & 2 & $\ldots$ & 58.376 & 0.019 \\
& 4 & $\ldots$ & 2 & 58.321 & 0.074 \\
\hline
\end{tabular}

a Plus : 118 core $+8 s^{2} 8 p^{n} 5 g^{18} 6 f^{14}$.

et al. ${ }^{10}$ to calculate the total energy. We have found new unexpected ground state configurations for E159 and E160. The valence electrons of E159 are expected to be $7 d^{4} 9 s^{1}$ and for E160, to be $7 d^{5} 9 s^{1}$ instead of the continuous filling of the $d$ shell $\left(7 d_{3 / 2}\right)^{4}\left(7 d_{5 / 2}\right)^{n}$ which was expected earlier. In Table I the total energies, $E_{T}$, and differences of the lowest lying electron configurations are listed for both elements. The very small difference, $\Delta E_{T}$, between the two lowest lying configurations for E160 makes this result questionable but the first difference for $\mathbf{E} 159$ is quite large. Already at element E161 the pure d electronic shell has increased stability so that the occupation of $9 s$ electronic states again disappear. The $9 s$ orbitals will start to fill again at E165 and E166 but from then on they remain occupied in all following atoms.

The filling of the shells in this region of elements is extremely unpredictable from an extrapolation based on the pattern of the filling in the known part of the periodic table. The increasing direct relativistic effect which can be mainly seen in (a) the increased bonding of the $s$ and $p_{1 / 2}$ electrons which results in a relatively. large probability density near the nucleus and (b) the increasing splitting of all shells into subshells with $j=l \pm \frac{1}{2}$. These are the two main effects which establish new criteria for the continuation of the periodic table. The early filling of the $9 s$ electrons which occurs between the completion of the $7 d_{3 / 2}$ subshell (which is full at E158) and the beginning of the $7 d_{5 / 2}$ shell is related to both effects. From this point of view, one should anticipate that after the first $9 s$ electron is favored in E159, a second $9 s$ electron would be added in at E160. The Coulomb interaction between the first and the second $9 s$ electron decreases the binding of both. At the same time, the binding of the $7 d_{5 / 2}$ rapidly increases with $Z$ and exchange favors binding for $d$ electrons more than for $s$ electrons. In the delicate balance, as indicated by the small values of $\Delta E_{T}$, the occupation of the $7 d_{\mathrm{j} / 2}$ orbital occurs at the expense of the second $9 s$ electron. At E161 the increased binding of the $d$ electrons is sufficient to "drain" the $9 s$ orbital. Therefore, one finds a pure $d$ configuration again. That is, the valence electrons of the ground state involve both $d$ and $s$ electrons just as ther do in the $d$-transition metals. Formally the situations are similar but the reasons are different.

* Supported by a grant from the U.S. Atomic Energy Commission.

'J. Grumann, U. Mosel, B. Fink, and W. Greiner, Z. Physik $228,371(1969)$.

${ }^{2}$ B. Fricke and W. Greiner, Phys. Letters 30 B, 317 (1969).

${ }^{3} \mathrm{~J}$. B. Mann, Proceedings of the Robert A. Welch Foundation Conference, XIII Transuranium Llements, Mendeleev Centennial, Houston, Texas, November 1969 , p. 430.

${ }^{4}$ B. Fricke, W. Greiner, and J. T. Waber, Theor. Chim. Acta 21, 235 (1971)

${ }^{5}$ R. A. Penneman, J. B. Mann, and C. K. Jorgensen, Chem. Phys. Letters 8, 321 (1971).

${ }^{\circ}$ B. liricke and J. T. Waber, Actinides Rev. 1, 433 (1971).

' B. liricke and J. T. Waber, J. Chem. Phys. (to be published).

${ }^{8}$ J. Grumann, T. Morovic, and $W$. Greiner (unpublished).

${ }^{9}$ D. Liberman, J. T. Waber, and D. T. Cromer, Phys. Rev. 137, A27 (1965); D. Liberman, D. T. Cromer, and J. T. Waber, Comp. Phys. Commun. 2, 107 (1971)

${ }^{10}$ E. C. Snow, J. Canfield, and J. T. Waber, Phys. Rev. 135, A969 (1964). 